\title{
THE DIEDERICH-FORNÆSS EXPONENT AND NON-EXISTENCE OF STEIN DOMAINS WITH LEVI-FLAT BOUNDARIES
}

\author{
SIQI FU AND MEI-CHI SHAW
}

\begin{abstract}
We study the Diederich-Fornæss exponent and relate it to non-existence of Stein domains with Levi-flat boundaries in complex manifolds. In particular, we prove that if the Diederich-Fornæss exponent of a smooth bounded Stein domain in an $n$-dimensional complex manifold is $>k / n$, then it has a boundary point at which the Levi-form has rank $\geq k$.
\end{abstract}

Mathematics Subject Classification (2010): 32T35, 32V40.

\section{INTRODUCTION}

A domain in a complex manifold is Stein if and only if there exists a smooth strictly plurisubharmonic exhaustion function. A Stein domain is called hyperconvex if there exists a smooth bounded strictly plurisubharmonic function. Diederich and Fornæss [11] showed that for any bounded pseudoconvex domain in $\mathbb{C}^{n}$ with $C^{2}$ boundary, there exist a positive constant $\eta$ and a defining function $\rho$ such that $\hat{\rho}=-(-\rho)^{\eta}$ is plurisubharmonic on $\Omega$ (see also [29]). The existence of bounded plurisubharmonic function was later generalized to bounded pseudoconvex domains with $C^{1}$ boundary by Kerzman and Rosay [20] and with Lipschitz boundary by Demailly [10] (see also more recent results by Harrington [15]). The constant $\eta$ is called a Diederich-Fornæss exponent. The supremum of all Diederich-Fornæss exponents is called the Diederich-Fornæss index of $\Omega$. The Diederich-Fornæss index has implications in regularity theory of the $\bar{\partial}$-Neumann Laplacian (see, for example, 21, 3, 5, 16]), as well as in estimates of the pluri-complex Green function [4] and comparison of the Bergman and Szegö kernels [8]. The Diederich-Fornæss indices can be arbitrarily small on the worm domains ([11, 12]). Sibony proved that for a smooth bounded pseudoconvex domain in $\mathbb{C}^{n}$ satisfying Property $(P)$, the Diederich-Fornæss index is one (see [31]).

If the pseudoconvex domain $\Omega$ has a defining function which is bounded plurisubharmonic on $\bar{\Omega}$, then the Diederich-Fornæss index is one. Fornæss and Herbig [13] showed that a smooth bounded domain in $\mathbb{C}^{n}$ with a defining function that is plurisubharmonic on the boundary also has Diederich-Fornæss index one. In this case, Boas and Straube showed that the $\bar{\partial}$-Neumann Laplacian is global hypoelliptic on $L^{2}$-Sobolev spaces (see [1]). It was shown by Nemirovskii [22, Corollary] that any smooth bounded Stein domain with a defining function that is plurisubharmonic on the domain cannot have Levi-flat boundary. In this paper, we study the Diederich-Fornæss exponent and relate it to non-existence of Stein domains with Levi-flat boundaries in complex manifolds. Our main result can be stated as follows:

Theorem 1.1. Let $\Omega$ be a bounded Stein domain with $C^{2}$ boundary in a complex manifold $M$ of dimension $n$. If the Diederich-Forncess index of $\Omega$ is greater than $k / n, 1 \leq k \leq n-1$, then $\Omega$ has a boundary point at which the Levi form has rank greater than $k$.

The authors were supported in part by NSF grants. 
In particular, we have the following corollary.

Corollary 1.2. If the Diederich-Forcess index is greater than $1 / n$, then its boundary cannot be Levi flat; and if the Diederich-Forcess exponent is greater than $1-1 / n$, then its boundary must have at least one strongly pseudoconvex boundary point.

We would like to thank Professor Takeo Ohsawa who kindly informed us that similar results were obtained by Adachi and Brinkschulte independently using different methods [37. For related work on the nonexistence of Levi-flat hypersurfaces in complex manifolds, we refer to the reader to papers [18, 22, 32, 33, 23, 5, 6, 25, 26] in the references.

\section{The Diederich-FornÆss IndeX}

Let $M$ be an $n$-dimensional complex manifold with hermitian metric $\omega$. Let $\Omega$ be a bounded domain in $M$. A continuous real-valued function $r$ on $M$ is called a defining function of $\Omega$ if $r<0$ on $\Omega, r>0$ on $M \backslash \bar{\Omega}$, and $C_{1} \delta(z) \leq|r(z)| \leq C_{2} \delta(z)$ near $b \Omega$, where $\delta(z)$ is the geodesic distance from $z$ to the boundary $b \Omega$. We will also assume that the defining function $r$ is in the same smoothness class as that of the boundary $b \Omega$. A defining function $r$ is said to be normalized if $\lim _{z \rightarrow b \Omega}|r(z)| / \delta(z)=1$. Note that the signed distance function $\rho(z)=-\delta(z)$ on $\Omega$ and $\rho(z)=\delta(z)$ on $M \backslash \Omega$ is a normalized defining function for $\Omega$.

A constant $0<\eta \leq 1$ is called a Diederich-Forncess exponent of a defining function $r$ of $\Omega$ if there exists a neighborhood $U$ of $b \Omega$ such that

$$
\partial \bar{\partial}\left(-(-r)^{\eta}\right) \geq 0
$$

on $U \cap \Omega$ in the sense of distribution. We will call the supremum of all such $\eta$ 's the DiederichForncess index of $r$ and denote it by $I(r)$. The supremum of $I(r)$ over all defining functions of $\Omega$ is called the Diederich-Forncess index of $\Omega$ and is denoted by $I(\Omega)$.

A defining function $r$ is said to satisfy the strong Oka property if there exists a constant $K$ and a neighborhood $U$ of $b \Omega$ such that

$$
\partial \bar{\partial}(-\log (-r)) \geq K \omega
$$

on $U \cap \Omega$ in the sense of distribution. The supremum of all such $K$ 's is called the $O k a$ index of $r$ and is denoted by $K(r)$. By Takeuchi's theorem, the signed distance function of a (proper) pseudoconvex domain in $\mathbb{C P}^{n}$ with the Fubini-Study metric satisfies the strong Oka property with Oka index 1/12. (Hereafter, the Fubini-Study metric is normalized so that its holomorphic sectional curvature is 2 and hence its holomorphic bisectional curvature is $\geq 1$.)

Let $\Omega \subset \subset M$ be a bounded domain with $C^{2}$-boundary. Let $r$ be a defining function of $\Omega$. Let $\omega_{\nu}=\partial r /|\partial r|$. Let $L_{\nu}$ be the dual vector of $\omega_{\nu}$. For any $(1,0)$-vector $X$ near $b \Omega$, let $X_{\nu}=\left\langle X, L_{\nu}\right\rangle_{\omega} L_{\nu}$ be the complex normal component of $X$ and $X_{\tau}=X-X_{\nu}$ the complex tangential component. Write $T^{1,0}(r)=\left\{(z, X) \in T^{1,0}(M) \mid X r=0\right\}$. For $z \in b \Omega$, we further decompose $X_{\tau}=X_{s}+X_{l}$, where $X_{l}$ is in the the null space $\mathcal{N}_{z}$ of the Levi-form $\partial \bar{\partial} \delta$ at $z$ and $X_{s} \perp X_{l}$. Let $S^{1,0}(M)=\left\{(z, X) \in T^{1,0}(M),|X|_{\omega}=1\right\}$. Let $W$ be the weakly pseudoconvex points on $b \Omega$. Let

$$
S(r)=\max \left\{\left|\partial \bar{\partial} r\left(X_{l}, \bar{L}_{\nu}\right)(z)\right| ; \quad\left|X_{l}\right|_{\omega}=1, X_{l} \in \mathcal{N}_{z}, z \in W\right\} .
$$

If $b \Omega$ is strongly pseudoconvex, we set $S(r)=0$. Define

$$
I_{0}(r)=\max \left\{\min \left\{\frac{K(r)}{8(S(r))^{2}}, \frac{1}{2}\right\}, 1-\frac{2(S(r))^{2}}{K(r)}\right\}>0 .
$$


Theorem 2.1. Let $\Omega$ be a bounded domain with $C^{2}$-boundary and let $r$ be a normalized defining function that satisfies the strong Oka property. Then $I(r) \geq I_{0}(r)$.

Proof. A simple computation yields that

$$
\partial \bar{\partial}(-\log (-r))=\frac{\partial \bar{\partial} r}{-r}+\frac{\partial r \wedge \bar{\partial} r}{r^{2}}
$$

and

$$
\begin{aligned}
\partial \bar{\partial}\left(-(-r)^{\eta}\right) & =\eta(-r)^{\eta}\left(\frac{\partial \bar{\partial} r}{-r}+(1-\eta) \frac{\partial r \wedge \bar{\partial} r}{r^{2}}\right) \\
& =\eta(-r)^{\eta}\left(\partial \bar{\partial}(-\log (-r))-\eta \frac{\partial r \wedge \bar{\partial} r}{r^{2}}\right) .
\end{aligned}
$$

It follows from (2.5) that (2.1) is equivalent to

$$
\partial \bar{\partial}(-\log (-r)) \geq \eta \frac{\partial r \wedge \bar{\partial} r}{r^{2}} .
$$

Let $c_{0}$ be a constant such that $0<c_{0}<K(r)$. Then

$$
\partial \bar{\partial}(-\log (-r)) \geq c_{0} \omega
$$

for $z \in \Omega$ near the boundary. It follows from (2.4) that

$$
\frac{\partial \bar{\partial} r\left(X_{\tau}, \bar{X}_{\tau}\right)}{-r} \geq c_{0}\left|X_{\tau}\right|_{\omega}^{2} \text {. }
$$

Let $C_{1}$ be any constant such that $C_{1}>S(r)$. Then there exists a neighborhood $U$ of $\mathcal{N}^{1,0}(W)=\left\{\left.(z, X)\left|z \in W, X \in \mathcal{N}_{z},\right| X\right|_{\omega}=1\right\}$ in $S^{1,0}(M)$ such that

$$
\left|\partial \bar{\partial} r\left(X, \bar{L}_{\nu}\right)\right| \leq C_{1}, \quad(z, X) \in U .
$$

For $\left(z, X_{\tau}\right) \in S^{1,0}(\bar{\Omega}) \backslash U$ with $z$ near $b \Omega$,

$$
\partial \bar{\partial} r\left(X_{\tau}, \bar{X}_{\tau}\right) \geq C_{2}\left|X_{\tau}\right|_{\omega}^{2}
$$

for some constant $C_{2}>0$. We write $X=X_{\tau}+X_{\nu}$ with $X_{l} \in \mathcal{N}_{z}$ as before. Then

$$
\begin{aligned}
\partial \bar{\partial}(-\log (-r))(X, \bar{X}) & =\frac{\partial \bar{\partial} r\left(X_{\tau}, \bar{X}_{\tau}\right)}{-r}+\frac{\partial \bar{\partial} r\left(X_{\nu}, \bar{X}_{\nu}\right)}{-r} \\
& +\frac{2 \operatorname{Re} \partial \bar{\partial} r\left(X_{\tau}, \bar{X}_{\nu}\right)}{-r}+\frac{|X r|^{2}}{r^{2}} .
\end{aligned}
$$

Note that $|X r|=\left|X_{\nu}\right|_{\omega} \cdot|\partial r|_{\omega}$. Let $K_{0}=\sup \left\{|\partial \bar{\partial} r|_{\omega} ; z \in \bar{\Omega}\right\}$. Then

$$
\left|\partial \bar{\partial} r\left(X_{\nu}, X_{\nu}\right)\right| \leq K_{0}|X r|^{2} /|\partial r|_{\omega}^{2}
$$

Similarly,

$$
\left|\operatorname{Re} \partial \bar{\partial} r\left(X_{\tau}, \bar{X}_{\nu}\right)\right| \leq K_{0}\left|X_{\tau}\right|_{\omega} \cdot|X r| /|\partial r|_{\omega} .
$$

We first deal with the strictly pseudoconvex directions. For $(z, X) \in T^{1,0}(\Omega)$ with $\left(z, X_{\tau} /\left|X_{\tau}\right|\right) \in S^{1,0}(\Omega) \backslash U$ with $z$ near $b \Omega$, it follows from (2.13) and (2.10) that for any positive constant $M$,

$$
\begin{aligned}
\left|2 \operatorname{Re} \partial \bar{\partial} r\left(X_{\tau}, \bar{X}_{\nu}\right)\right| & \leq K_{0}\left(\frac{1}{M}\left|X_{\tau}\right|_{\omega}^{2}+\frac{M}{|\partial r|_{\omega}^{2}}|X r|^{2}\right) \\
& \leq \frac{K_{0}}{M C_{2}} \partial \bar{\partial} r\left(X_{\tau}, \bar{X}_{\tau}\right)+\frac{K_{0} M}{|\partial r|_{\omega}^{2}}|X r|^{2} .
\end{aligned}
$$


Therefore,

$$
\begin{aligned}
\partial \bar{\partial}(-\log (-r))(X, \bar{X}) \geq(1- & \left.\frac{K_{2}}{M C_{2}}\right) \frac{\partial \bar{\partial} r\left(X_{\tau}, \bar{X}_{\tau}\right)}{-r} \\
& +\left(1-\frac{K_{0}(M+1)|r|}{|\partial r|_{\omega}^{2}}\right) \frac{|X r|^{2}}{r^{2}} .
\end{aligned}
$$

By choosing $M$ sufficiently large and then letting $z$ be sufficiently close to $b \Omega$, we know that (2.6) holds for any $\eta<1$.

We now deal with weakly pseudoconvex directions. For $(z, X) \in T^{1,0}(\Omega)$ with $\left(z, X_{\tau} /\left|X_{\tau}\right| \omega\right) \in$ $U$, we have

$$
2\left|\partial \bar{\partial} r\left(X_{\tau}, \bar{X}_{\nu}\right)\right| \leq 2 C_{1}\left|X_{\tau}\right|_{\omega}|X r| /|\partial r|_{\omega} \leq C_{1}\left(\frac{|r|}{\varepsilon}\left|X_{\tau}\right|_{\omega}^{2}+\frac{\varepsilon}{|r|} \frac{|X r|^{2}}{|\partial r|_{\omega}^{2}}\right),
$$

where $\varepsilon$ is a positive constant to be chosen. Since $r$ is a normalized defining function, $|\partial r|_{\omega}=1 / \sqrt{2}$ on $b \Omega$. Combining (2.16) with (2.8), we have

$$
\begin{aligned}
\partial \bar{\partial}(-\log (-r))(X, \bar{X}) & \geq\left(c_{0}-C_{1} / \varepsilon\right)\left|X_{\tau}\right|^{2}+\frac{1-\left(C_{1} \varepsilon+K|r|\right)|\partial r|_{\omega}^{-2}}{r^{2}}|X r|^{2} \\
& \geq\left(c_{0}-C_{1} / \varepsilon\right)\left|X_{\tau}\right|^{2}+\frac{1-2 C_{1} \varepsilon-K^{\prime}|r|}{r^{2}}|X r|^{2}
\end{aligned}
$$

for some positive constant $K^{\prime}$.

We consider two cases: $4 C_{1}^{2} \leq c_{0}$ and $4 C_{1}^{2}>c_{0}$. When $4 C_{1}^{2} \leq c_{0}$, we take $\varepsilon=C_{1} / c_{0}$. Then

$$
\partial \bar{\partial}(-\log (-r))(X, \bar{X}) \geq\left(1-2 C_{1}^{2} / c_{0}-K^{\prime}|r|\right)|X r|^{2} / r^{2} .
$$

When $4 C_{1}^{2}>c_{0}$, we take $\varepsilon=\frac{1}{4 C_{1}}<C_{1} / c_{0}$. Then combining (2.17) with (2.7), we have

$$
\partial \bar{\partial}(-\log (-r))(X, \bar{X}) \geq-\left(\frac{C_{1}}{c_{0} \varepsilon}-1\right) \partial \bar{\partial}(-\log (-r))(X, \bar{X})+\frac{1-2 C_{1} \varepsilon-K^{\prime}|r|}{r^{2}}|X r|^{2} .
$$

Therefore,

Hence

$$
\partial \bar{\partial}(-\log (-r))(X, \bar{X}) \geq\left(\frac{c_{0} \varepsilon\left(1-2 C_{1} \varepsilon\right)}{C_{1}}-\frac{K^{\prime} c_{0} \varepsilon|r|}{C_{1}}\right) \frac{|X r|^{2}}{r^{2}}
$$

$$
\partial \bar{\partial}(-\log (-r))(X, \bar{X}) \geq\left(\frac{c_{0}}{8 C_{1}^{2}}-\frac{K^{\prime} c_{0} \varepsilon|r|}{2 C_{1}^{2}}\right) \frac{\left|X_{\nu}\right|^{2}}{r^{2}} .
$$

Note that when $4 C_{1}^{2} \leq c_{0}$, we have

$$
1-\frac{C_{1}^{2}}{c_{0}} \geq \frac{1}{2} \text { and } \frac{c_{0}}{4 C_{1}^{2}} \geq \frac{1}{2}
$$

Furthermore, when $4 C_{1}^{2}>c_{0}$,

$$
\frac{1}{2}>\frac{c_{0}}{8 C_{1}^{2}}>1-\frac{C_{1}^{2}}{c_{0}}
$$

Combing (2.18)-(2.21), we know that (2.6) holds for any $\eta<I_{0}(r)$. We thus conclude the proof of Proposition 2.1

By Takeuchi's theorem ([36], see also [7, 14]), (2.2) holds for the signed distance function with $K=1 / 12$ on any proper pseudoconvex domain on complex projective space $\mathbb{C P}^{n}$. Combing this with Proposition 2.1, we have: 
Corollary 2.2. Let $\Omega$ be a proper pseudoconvex domain in $\mathbb{C P}^{n}$ with $C^{2}$ boundary. Then its Diederich-Fornaess index

$$
I(\Omega) \geq I_{0}(\rho)=\max \left\{\min \left\{\frac{1}{96(S(\rho))^{2}}, \frac{1}{2}\right\}, 1-24(S(\rho))^{2}\right\}>0,
$$

where $\rho$ is the signed distance function to $b \Omega$ with respect to the Fubini-Study metric.

Proposition 2.3. Let $\Omega \subset \subset M$ be a bounded domain with $C^{2}$ boundary and let $r$ be a normalized defining function. Suppose (2.2) holds and there exist a neighborhood $V$ of the set $W$ of weakly pseudoconvex boundary points and a positive constant $K_{1}>1$ such that

$$
K\left|X_{\tau}\right|^{2} \leq \frac{\partial \bar{\partial} r\left(X_{\tau}, \bar{X}_{\tau}\right)}{r} \leq K K_{1}\left|X_{\tau}\right|^{2}
$$

for all $z \in V$ and $X \in T_{z}^{1,0}(M)$. Then

$$
I(\Omega) \geq \max \left\{\min \left\{\frac{1}{8\left(K_{1}-1\right)}, \frac{1}{2}\right\}, 3-2 K_{1}\right\} .
$$

Proof. From (2.2), we know that

$$
\Theta=\partial \bar{\partial}(-\log (-r))-K \omega
$$

is positive semi-definite. Applying the Cauchy-Schwarz inequality to $\Theta\left(X_{\tau}, X_{\nu}\right)$, we then have

$$
\left|\Theta\left(X_{\tau}, X_{\nu}\right)\right| \leq\left|\Theta\left(X_{\tau}, X_{\tau}\right)\right|^{1 / 2}\left|\Theta\left(X_{\nu}, X_{\nu}\right)\right|^{1 / 2} .
$$

(We refer the reader to [34] for a similar technique that has been used by Straube to construct Stein neighborhood bases in connection with regularity theory in the $\bar{\partial}$-Neumann problem.) Therefore,

$$
\left|\frac{\partial \bar{\partial} r\left(X_{\tau}, \bar{X}_{\nu}\right)}{r}\right|^{2} \leq\left(\frac{\partial \bar{\partial} r\left(X_{\tau}, X_{\tau}\right)}{-r}-K\left|X_{\tau}\right|_{\omega}^{2}\right)\left(\frac{\partial \bar{\partial} r\left(X_{\nu}, X_{\nu}\right)}{-r}+\frac{|X r|^{2}}{r^{2}}-K\left|X_{\nu}\right|_{\omega}^{2}\right) .
$$

Thus

$$
\left|\partial \bar{\partial} r\left(X_{\tau}, \bar{X}_{\nu}\right)\right| \leq\left(\left(K_{1}-1\right) K\right)^{1 / 2}(1+C|r|)^{1 / 2}\left|X_{\tau}\right|\left|X_{\nu}\right|
$$

for some positive constant $C$. The inequality (2.23) then follows by applying Proposition 2.1 with $S(r)=\left(\left(K_{1}-1\right) K\right)^{1 / 2}$.

Let $f \in C^{2}(M)$. Recall that the real Hessian $H_{f}$ is defined by

$$
H_{f}(\xi, \zeta)(z)=\left\langle\nabla_{\xi}(\nabla f), \zeta\right\rangle
$$

for $\xi, \zeta \in T_{\mathbb{R}}\left(M^{2 n}\right)$, where $\nabla_{\xi}$ denotes the covariant derivative. For any $X \in T_{\mathbb{C}}^{1,0}(M)$, we write $X=\frac{1}{\sqrt{2}}\left(\xi_{X}-\sqrt{-1} J \xi_{X}\right)$ where $J$ is the complex structure. Let $z$ be a point in $\Omega$ near the boundary and $\pi(z)$ be its closest point on $b \Omega$. Let $\gamma(t)$ be the geodesic parametrized by arc-length such that $\gamma(0)=\pi(z)$. For any $(1,0)$ tangent vector $X$ at $z$ near $b \Omega$, we let $X(t)$ be the vector at $\gamma(t)$ obtained by parallel translate (of real and imaginary parts) of $X$ along the geodesic from $z$ to $\gamma(t)$ and let $X^{0}=X(0)$.

Proposition 2.4. Let $\Omega$ be a proper pseudoconvex domain with $C^{2}$ boundary in $\mathbb{C P}^{n}$. Let $\rho$ be the signed distance function to $b \Omega$ with respect to the Fubini-Study metric. Let

$$
K_{2}=\max \left\{\left|\nabla_{\xi_{X}}(\nabla \rho)\right|_{\omega}^{2}+\left|\nabla_{J \xi_{X}}(\nabla \rho)\right|_{\omega}^{2} ; z \in W, X \in \mathcal{N}_{z},|X|_{\omega}=1\right\}
$$

Then

$$
I(\Omega) \geq \max \left\{\min \left\{\frac{1}{8\left(K_{2}-1\right)}, \frac{1}{2}\right\}, 3-2 K_{2}\right\}
$$


Proof. It follows from the computations in [35] that

$$
\lim _{t \rightarrow 0^{+}} \frac{1}{t}\left(\partial \bar{\partial} \rho\left(X_{\tau}(t), X_{\tau}(t)\right)-\partial \bar{\partial} \rho\left(X^{0}, \bar{X}^{0}\right)\right)=\left|\nabla_{\xi_{X}}(\nabla \rho)\right|_{\omega}^{2}+\left|\nabla_{J \xi_{X}}(\nabla \rho)\right|_{\omega}^{2}
$$

(The above identity was proved in [35] for $\Omega$ in $\mathbb{C}^{n}$; compare also [34]. The proof for $\Omega$ in $\mathbb{C P}^{n}$ is similar; see 7 for related arguments.) We then conclude the proof by applying Proposition 2.3 with $K=1$ and any $K_{1}>K_{2}$.

From Proposition 2.1, we also obtain the following slight variation of a result of Ohsawa and Sibony ([27]; see also [5, 6]):

Corollary 2.5. Let $\Omega$ be a bounded domain in $M$ with $C^{2}$ boundary. Suppose $r$ is a normalized defining function that satisfies (2.2). Then for any $c \in(0, K)$ and $\eta \in\left(0, I_{0}(r)\right)$, there exists a neighborhood $V$ of $b \Omega$ such that

$$
\partial \bar{\partial}(-\log (-r)) \geq c \omega+\left(1-\frac{c}{K}\right) \eta \frac{\partial r \wedge \bar{\partial} r}{r^{2}}
$$

and

$$
\partial \bar{\partial}\left(-(-r)^{\eta}\right) \geq \eta(-r)^{\eta}\left(c \omega+\left(1-\frac{c}{K}\right) \eta \frac{\partial r \wedge \bar{\partial} r}{r^{2}}\right) .
$$

\section{Non-existence of Stein domains with Levi-flat boundaries}

We prove Theorem 1.1 in this section. We first recall the following well-known simple lemma. Let $\Omega$ be a bounded domain with $C^{2}$ boundary in a complex hermitian manifold $M$ of dimension $n$. Let $\rho$ be a defining function for $\Omega$. For $t>0$, let $\Omega_{-t}=\{z \in \Omega ; \rho<-t\}$. Let $i_{t}: b \Omega_{-t} \rightarrow M$ be the inclusion map. Let $1 \leq k \leq n$ be an integer.

Lemma 3.1. If the rank of the Levi form of $b \Omega i s \leq k-1$ at all $z \in b \Omega$, then

$$
i_{t}^{*}\left(d^{c} \rho \wedge\left(d d^{c} \rho\right)^{n-1}\right)=O\left(t^{n-k}\right) d S_{t}
$$

where $d S_{t}$ is the surface element of $b \Omega_{-t}$.

We sketch the proof for the reader's convenience. Note that $d S_{t}=i_{t}^{*}(* d r) /|d r|_{\omega}$ and

$$
\left.i_{t}^{*}\left(d^{c} \rho \wedge\left(d d^{c} \rho\right)^{n-1}\right)=\nu\right\lrcorner\left((d \rho /|d \rho|) \wedge d^{c} \rho \wedge\left(d d^{c} \rho\right)^{n-1}\right)
$$

where $\nu$ is the dual vector of $d \rho /|d \rho|_{\omega}$. By choosing local holomorphic coordinates that diagonalize the Levi form, we then obtain (3.1).

We now prove Theorem 1.1. Let $\rho$ be a defining function of $\Omega$ such that $\hat{\rho}=-(-\rho)^{\eta}$ is plurisubharmonic on $\Omega$ for some constant $\eta>k / n$. Let $\Omega_{-t}=\{\rho<-t\}, t>0$. Since $\Omega$ is Stein, $\Omega_{-t}$ has at least a strictly pseudoconvex boundary point for sufficiently small $t$. Let

$$
f(t)=\int_{\Omega_{-t}}\left(d d^{c} \hat{\rho}\right)^{n}
$$

Then $f(t) \geq 0$ and $f(t)$ is decreasing. By Stokes' theorem,

$$
f(t)=\int_{b \Omega_{-t}} i_{t}^{*}\left(d^{c} \hat{\rho} \wedge\left(d d^{c} \hat{\rho}\right)^{n-1}\right) .
$$

Since

$$
d^{c} \hat{\rho}=i \eta(-\rho)^{\eta-1}(\bar{\partial} \rho-\partial \rho) \quad \text { and } \quad d d^{c} \hat{\rho}=2 i \eta \rho^{\eta}\left(\frac{\partial \bar{\partial} \rho}{-\rho}+(1-\eta) \frac{\partial \rho \wedge \bar{\partial} \rho}{\rho^{2}}\right),
$$

we have

$$
d^{c} \hat{\rho} \wedge\left(d d^{c} \hat{\rho}\right)^{n-1}=\eta^{n}(-\rho)^{n(\eta-1)} d^{c} \rho \wedge\left(d d^{c} \rho\right)^{n-1} .
$$


Suppose the Levi rank of $b \Omega$ is $\leq k-1$ at all boundary points, then by Lemma 3.1 ,

$$
i_{t}^{*}\left(d^{c} \rho \wedge\left(d d^{c} \rho\right)^{n-1}\right)=O\left(t^{n-k}\right) d S_{t} .
$$

Thus

$$
f(t)=O\left(t^{n \eta-k}\right)
$$

Therefore, $\lim _{t \rightarrow 0^{+}} f(t)=0$ and hence $f(t)=0$ for small $t>0$. This implies that $b \Omega_{-t}$ has Levi rank $\leq n-2$ at each point, which leads to a contradiction. This concludes the proof of Theorem 1.1.

Corollary 1.2 follows easily. The following theorem is a variation of Theorem 1.1.

Theorem 3.2. Let $M$ be a complex manifold of dimension $n$ with a hermitian metric $\omega$. Let $\Omega$ be a bounded Stein domain in $M$ with $C^{2}$ boundary. Suppose there exist a defining function $\rho$, a constant $\eta>0$, and a neighborhood $U$ of $b \Omega$ such that

$$
\partial \bar{\partial}\left(-(-\rho)^{\eta}\right) \geq c(-\rho)^{\eta}\left(\omega+\frac{\partial \rho \wedge \bar{\partial} \rho}{\rho^{2}}\right)
$$

on $U \cap \Omega$ for some constant $c>0$. If $\eta \geq 1 / n$, then $\Omega$ cannot have Levi-flat boundary.

Proof. In light of Theorem 1.1, it remains to prove the case when $\eta=1 / n$. We follow the notations as in the above proof of Theorem [1.1. Let $\varepsilon_{0}$ be sufficiently small such that $\Omega \backslash \Omega_{-\varepsilon_{0}} \subset U \cap \Omega$. We set

$$
f(t)=\int_{\Omega_{-t} \backslash \Omega_{-\varepsilon_{0}}}\left(d d^{c} \widehat{\rho}\right)^{n}
$$

for $0<t<\varepsilon_{0}$. Suppose $b \Omega$ is Levi-flat, then as in the proof of Theorem 1.1.

$$
\left.d^{c} \hat{\rho} \wedge\left(d d^{c} \hat{\rho}\right)^{n-1}\right|_{b \Omega_{-t}}=\left.\eta^{n}(-\rho)^{n(\eta-1)} d^{c} \rho \wedge\left(d d^{c} \rho\right)^{n-1}\right|_{b \Omega_{-t}}=O\left(t^{n \eta-1}\right) d S_{t} \leq C d S_{t} .
$$

By Stoke's theorem

$$
f(t)=\int_{b \Omega_{-t}} d^{c} \hat{\rho} \wedge\left(d d^{c} \hat{\rho}\right)^{n-1}-\int_{b \Omega_{-\varepsilon_{0}}} d^{c} \hat{\rho} \wedge\left(d d^{c} \hat{\rho}\right)^{n-1} \leq C .
$$

On the other hand, it follows from (3.2) that

$$
\left(d d^{c} \widehat{\rho}\right)^{n} \geq C \delta^{n \eta}\left(\omega+\frac{\partial \delta \wedge \bar{\partial} \delta}{\delta^{2}}\right)^{n} \geq C \delta^{n \eta-2} d V
$$

where $d V$ is the volume element. Thus

$$
\begin{aligned}
f(t) & =\int_{\Omega_{-t} \backslash \Omega_{-\varepsilon_{0}}}\left(d d^{c} \hat{\rho}\right)^{n} \geq C \int_{\Omega_{-t} \backslash \Omega_{-\varepsilon_{0}}}(-\rho)^{n \eta-2} d V \\
& \geq C \int_{-\varepsilon_{0}}^{-t}(-\rho)^{-1} d \rho \geq C\left(-\log t+\log \varepsilon_{0}\right) .
\end{aligned}
$$

Therefore, $\lim _{t \rightarrow 0^{+}} f(t)=\infty$, which leads to a contradiction with (3.3). This concludes the proof of Proposition 3.2 .

Acknowledgement: This work was done while the first author visited the University of Notre Dame in April, 2012. He thanks the Department of Mathematics for the warm hospitality. 


\section{REFERENCES}

[1] H. P. Boas and E. J. Straube, Sobolev estimates for the $\bar{\partial}$-Neumann operator on domains in $\mathbb{C}^{n}$ admitting a defining function that is plurisubharmonic on the boundary, Math. Z. 206 (1991), 8188.

[2] B. Berndtsson, Weighted estimates for the $\bar{\partial}$-equation, Complex Analysis and Complex Geometry (J. D. McNeal eds.), de Gruyter, pp. 43-57, 2001.

[3] B. Berndtsson and Ph. Charpentier, A Sobolev mapping property of the Bergman knernel, Math. Z. 235 (2000), 1-10.

[4] Z. Błocki, The Bergman metric and the pluricomplex Green function, Trans. Amer. Math. Soc. 357 (2004), 2613-2625.

[5] J. Cao, M.-C. Shaw, and L. Wang, Estimates for the $\bar{\partial}-$ Neumann problem and nonexistence of $C^{2}$ Levi-flat hypersurfaces in $\mathbb{C} P^{n}$, Math. Z. 248 (2004), 183-221. Erratum, 223-225.

[6] J. Cao and M.-C. Shaw, The $\bar{\partial}-$ Cauchy problem and nonexistence of Lipschitz Levi-flat hypersurfaces in $\mathbb{C} P^{n}$ with $n \geq 3$, Math. Z. 256 (2007), 175-192.

[7] _ A new proof of the Takeuchi Theorem, Lecture Notes of Seminario Interdisp. di Mate. 4 (2005), 65-72.

[8] B. Chen and S. Fu, Comparison of the Bergman and Szeg kernels, Advances in Mathematics 228 (2011), 2366-2384.

[9] J.-P. Demailly, Estimations $L^{2}$ pour l'opérateur $\bar{\partial}$ d'un fibré vectoriel holomorphe semi-positif audessus d'une variété kählérienne complète, Ann. Sci. École Norm. Sup. 15 (1982), 457-511.

[10] _ Mesures de Monge-Ampere et mesures plurisousharmoniques, Math. Z. 194 (1987), 519-564.

[11] K. Diederich and J. E. Fornæss, Pseudoconvex domains: bounded strictly plurisubharmonic exhaustion functions, Invent. Math. 39 (1977), 129-141.

[12] _ Pseudoconvex domains: an example with nontrivial Nebenhülle, Math. Ann. 225 (1977), $275-292$.

[13] J. E. Fornæss and A.-K. Herbig, A note on plurisubharmonic defining functions in $\mathbb{C}^{n}$, Math. Ann. 342 (2008), 749-772.

[14] R. E. Greene and H. Wu, On Kähler manifolds of positive bisectional curvature and a theorem of Hartogs, Abh. Math. Sem. Univ. Hamburg 47 (1978), 171-185.

[15] P. S. Harrington, The order of plurisubharmonicity on pseudoconvex domains with Lipschitz boundaries, Math. Res. Lett. 14 (2007), 485-490.

[16] - Global regularity for the $\bar{\partial}-$ Neumann operator and bounded plurisubharmonic exhaustion functions, Adv. Math. 228 (2011), no. 4, 2522-2551.

[17] G. Henkin and A. Iordan, Regularity of $\bar{\partial}$ on pseudoconcave compacts and applications, Loo-Keng Hua: a great mathematician of the twentieth century. Asian J. Math. 4 (2000), no. 4, 855-883. Erratum, Asian J. Math. 7 (2003), no. 1, 147-148.

[18] A. Lins Neto, A note on projective Levi flats and minimal sets of algebraic foliations, Ann. Inst. Fourier 49(1999), 1369-1385.

[19] M. Klimek, Pluripotential theory, Oxford University Press, 1991.

[20] N. Kerzman and J.-P. Rosay, Fonctions plurisousharmoniques d'exhaustion bornées et domaines taut, Math. Ann. 257 (1981), no. 2, 171-184.

[21] J. J. Kohn, Quantitative estimates for global regularity, Analysis and geometry in several complex variables (Katata, 1997), 97-128, Trends Math., Birkhuser Boston, Boston, MA, 1999.

[22] S. Nemirovskii, Stein domains with Levi-plane boundaries on compact complex surfaces (Russian) Mat. Zametki 66 (1999), 632-635; translation in Math. Notes 66 (1999), 522-525.

[23] L. Ni and J. Wolfson, The Lefschetz theorem for CR submanifolds and the nonexistence of real analytic Levi flat submanifolds, Comm. Analysis and Geometry 11 (2003), 553-564.

[24] T. Ohsawa, Nonexistence of real analytic Levi flat hypersurfaces in $\mathbf{P}^{2}$, Nagoya Math. J. 158 (2000), 95-98.

[25] _ On the complement of Levi-flats in Khler manifolds of dimension $\geq 3$., Nagoya Math. J. 185 (2007), 161-169.

[26] - A survey on Levi flat hypersurfaces, preprint, 2013.

[27] T. Ohsawa and N. Sibony, Bounded P.S.H functions and pseudoconvexity in Kähler manifolds. Nagoya Math. J. 149 (1998), 1-8.

[28] T. Ohsawa and K. Takegoshi, On the extension of $L^{2}$ holomorphic functions, Math. Z. 195 (1987), 197-204. 
[29] R. M. Range: A remark on bounded strictly plurisubharmonic exhaustion functions, Proc. Amer. Math. Soc., 81 (1981), 220-222.

[30] R. Richberg, Stetige streng pseudokonvexe funktionen, Math. Ann. 175 (1968), 257-286.

[31] N. Sibony, Une classe de domaines pseudoconvexes, Duke Math. J. 55 (1987), 299-319.

[32] Y.-T. Siu, Nonexistence of smooth Levi-flat hypersurfaces in complex projective spaces of dimension $\geq 3$, Ann. of Math. 151 (2000), 1217-1243.

[33] —, $\bar{\partial}$-regularity for weakly pseudoconvex domains in compact Hermitian symmetric spaces with respect to invariant metrics, Ann. of Math. 156 (2002), 595-621.

[34] E. Straube, Good Stein neighborhood bases and regularity of the $\bar{\partial}$-Neumann problem, Illinois J. of Math. 45 (2001), 856-871.

[35] B. Weinstock, Some conditions for uniform H-convexity, Illinois J. Math. 19 (1975), 400-404.

[36] A. Takeuchi,Domaines pseudoconvexes infinis et la métrique riemannienne dans un espace projectif, J. Math. Soc. Japan 16(1964), 159-181.

[37] M. Adachi and J. Brinkschulte, A global estimate for the Diederich-Fornass index of weakly pseudoconvex domains, Preprint, 2014.

Department of Mathematical Sciences, Rutgers University-Camden, Camden, NJ 08102

E-mail address: sfu@camden.rutgers.edu

Department of Mathematics, University of Notre Dame, Notre Dame, IN 46556

E-mail address: Mei-Chi.Shaw.1@nd.edu 